\title{
Use of Dietary Supplements in Cancer: A Single-Institution Study
}

\author{
Shaghayegh Kamian (101, ${ }^{1,}$ and Ahmad R Mafi (i) ${ }^{1}$ \\ ${ }^{1}$ Radiation Oncology Department, Jorjani Cancer Center, Imam Hossein Educational Hospital, Shahid Beheshti University of Medical Sciences, Tehran, Iran \\ "Corresponding author: Radiation Oncology Department, Jorjani Cancer Center, Imam Hossein Educational Hospital, Shahid Beheshti University of Medical Sciences, Tehran, \\ Iran. Tel: +98-9123114846, Email: shkamian@sbmu.ac.ir
}

Received 2018 May 22; Revised 2018 November 21; Accepted 2018 December 18.

\begin{abstract}
Background: Many patients with cancer tend to use vitamin supplements alongside their conventional cancer treatments. They have the presumption that vitamins can help them feel better or fight the disease.

Objectives: The aim of this study was to determine the prevalence of vitamin use among cancer patients in a referral cancer center in Tehran, Iran.

Methods: Two hundred cancer patients who were undergoing outpatient chemotherapy or chemo-radiation were chosen. The data about the use of vitamins and whether they had been prescribed by their physician were collected by a questionnaire.

Results: Among the patients who were being treated with chemotherapy alone (81.5\% of the cases), $20.4 \%$ reported regular use of vitamins. This figure for the remaining $18.5 \%$ who were undergoing chemoradiation was $23.7 \%$. The interesting finding was that in $43.9 \%$ of the cases who were taking vitamins, it was prescribed by the treating oncologist. More than half ( $52.5 \%)$ of the patients who were taking vitamins had no other medical illness apart from cancer. Multivitamin capsules were the most commonly used form of vitamins.

Conclusions: More than $20 \%$ of our patients took vitamins regularly. In more than $40 \%$ of these cases, vitamin supplements had been prescribed by the treating physician. As there is some evidence that vitamins can interfere with chemotherapy and probably radiotherapy treatments, it would be prudent for oncologists to prescribe them more cautiously for the cancer patients who are under active treatment.
\end{abstract}

Keywords: Vitamins, Chemotherapy, Cancer Treatment, Dietary Supplements

\section{Background}

Apart from a few exceptions, such as vitamin D, the human body is not capable of synthesizing vitamins on its own in sufficient amounts and therefore, vitamins must mainly be obtained from the dietary intake.

There is emerging evidence from observational studies that different types of vitamin deficiency can be associated with development of chronic diseases such as atherosclerosis, cancer, and osteoporosis (1).

Despite the fact that there is no compelling evidence that any dietary supplement can help treat or prevent any type of cancer, it seems that many patients with cancer tend to use vitamin supplements alongside their conventional cancer treatments, such as radiotherapy or chemotherapy with the presumption that vitamins can help them feel better or fight the disease (2).

Interestingly, many of our patients who take regular vitamins and supplements tend to hide this information from their treating physician, unless they are asked di- rectly about the matter. This is more evident in patients who are referred for radiotherapy after completion the course of chemotherapy. Many of these patients take regular vitamins in order to reduce the symptoms or side effects of the treatment such as fatigue, nausea, anemia, etc.

There are, however, situations that vitamin intake seems necessary. For instance, many patients in the palliative setting, or patients who have had gastrectomy for gastric cancer need to take supplemental iron, vitamin B12, or many other types of vitamins and minerals.

While there is still controversy about whether vitamin supplements have any beneficial effect on cancer treatment, a growing number of evidences show that some vitamins can affect the efficacy of chemotherapy or radiation therapy. Therefore, if such drugs are misused, they may compromise the treatment results (3). 


\section{Objectives}

There is limited information about the use of nutritional supplements in cancer, and in our country, there is no available data for this issue. In this study, we evaluated the use of vitamin and nutritional supplements in cancer patients.

\section{Methods}

Between January and June 2017, two hundred cancer patients who were undergoing outpatient chemotherapy or chemoradiation at Jorjani Cancer Center and were willing to participate were chosen. In either adjuvant or metastatic setting, we selected the cases who were within three months of initiation of treatment, and all the patients filled the written consent form.

The data were collected by using an anonymous questionnaire. In that questionnaire, we filled the demographic characteristics of cases which included gender, age, level of education, marital status, the type of cancer and also metastatic versus non metastatic cancer, and the type of treatment (chemotherapy alone or chemoradiotherapy).

Demographic and cancer -related data, as well as data on vitamin supplement use were collected. We also asked whether they had made their own decision to take vitamins, or they had physician's prescription.

For practical reasons, based on the known popularity of various vitamin supplements among our people, we divided the used vitamins to three main categories: $B$ vitamins including B-complex (which contains all of the known essential water-soluble vitamins except for vitamin C), multivitamins, other drugs. Analysis was done with the version 22 of SPSS software.

\section{Results}

The majority of the patients were female (59.7\%) and $40.2 \%$ had attained at least a high school diploma. The demographic characteristics of the cases are presented in Table 1.

Overall, Forty-one of the patients (20.5\%) used vitamins and other supplements. Twenty cases used multivitamins and nine of them B vitamins (most commonly vitamin Bcomplex). Twelve patients used other supplements such as calcium, vitamin D, etc.

The use of vitamin supplements was seen more frequently in the patients who were in their sixth decade of life. Patients with higher education also had higher rates of

\begin{tabular}{|cc|}
\hline Table 1. The Demographic Characteristics of Patients & No. (\%) \\
\hline Variables & \\
\hline Gender & $81(40.5)$ \\
\hline Male & $119(59.5)$ \\
\hline Female & \\
\hline Education & $118(59)$ \\
\hline Under diploma & $51(25.8)$ \\
\hline Diploma & $31(84.8)$ \\
\hline University education & \\
\hline Marital status & $44(22)$ \\
\hline Single & $156(78)$ \\
\hline Married & $70(36.3)$ \\
\hline Metastasis & $123(63.7)$ \\
\hline Non-metastatic disease & $7(3.5)$ \\
\hline Metastatic disease & $162(81)$ \\
\hline Missing & $38(19)$ \\
\hline Chemotherapy & \\
\hline
\end{tabular}

vitamin consumption. Furthermore, single patients tend to use supplements more often than married ones $(48.1 \%$ vs. $20 \%$ ).

As it is shown in Table 2, the three most common cancers in our study were breast, head and neck and gynecologic cancers (as our center is a referral center for head and neck and gynecological cancers).

There were ten patients with gastric cancer, of which four of them had metastatic disease. One of these metastatic cases used multivitamin capsules that had not been prescribed by his physician. The other nine patients did not use any supplements. There were also six cases of cervical cancer, and two of them who had non metastatic disease used multivitamin prescribed by their physician.

Overall, 29 cases had anemia. Twenty of them did not use vitamin or iron supplements. Among the other nine (4 breast cancers, 2 endometrial cancers, 2 laryngeal cancers and one lung neoplasm), seven took vitamins prescribed by their physician, and the other two used vitamins without prescription.

More than one third (36.3\%) of the patients had metastatic disease. Vitamin consumption was more prevalent in these patient compared to non-metastatic cases (25.7\% vs. $18.7 \%$ ). These results are shown in Table 3.

Among the patients who were being treated with chemotherapy alone ( $81.5 \%$ of the cases), $20.4 \%$ reported 


\begin{tabular}{lc}
\hline Table 2. The Type of Cancer in Cases & \\
\hline Cancer & Number of Cases \\
\hline Breast & 60 \\
\hline Head and neck & 58 \\
\hline Gynecologic & 42 \\
\hline Gastrointestinal & 28 \\
\hline Sarcoma & 2 \\
\hline Lung & 6 \\
\hline Urologic & 3 \\
\hline Hodgkin lymphoma & 1 \\
\hline Total & 200 \\
\hline
\end{tabular}

regular use of vitamins. This figure for the remaining $18.5 \%$ who were undergoing chemoradiation was $23.7 \%$ (Table 4 ).

The majority of the patients (66\%) who were taking vitamins started using them after the second cycle of chemotherapy (Figure 1). There were six cases who had started supplements before chemotherapy for at least 3 months (three laryngeal, one ovary, one endometrial and one breast cancer).

The interesting finding was that in $43.9 \%$ of the cases were taking vitamins, it was prescribed by their treating oncologist. Weakness (22\%) and suggestion by family and friends (22\%) were other reasons for taking vitamins by the patients. More than half of the patients (52.5\%) who were taking vitamins had no other medical illness apart from cancer. We asked the patients about any symptom allevi-

\begin{tabular}{|c|c|c|c|}
\hline & \multicolumn{2}{|c|}{ Vitamin Use } & \multirow[t]{2}{*}{ Total } \\
\hline & Yes & No & \\
\hline \multicolumn{4}{|l|}{ Cancer } \\
\hline \multicolumn{4}{|l|}{ Metastatic } \\
\hline Count & 18 & 52 & 70 \\
\hline$\%$ within cancer & 25.7 & 74.3 & 100.0 \\
\hline$\%$ of total & 9.3 & 26.9 & 36.3 \\
\hline \multicolumn{4}{|l|}{ Non-metastatic } \\
\hline Count & 23 & 100 & 123 \\
\hline$\%$ within cancer & 18.7 & 81.3 & 100.0 \\
\hline$\%$ of total & 11.9 & 51.8 & 63.7 \\
\hline \multicolumn{4}{|l|}{ Total } \\
\hline Count & 41 & 152 & 193 \\
\hline$\%$ within cancer & 21.2 & 78.8 & 100.0 \\
\hline$\%$ of total & 21.2 & 78.8 & 100.0 \\
\hline
\end{tabular}

\begin{tabular}{|c|c|c|c|}
\hline & \multicolumn{2}{|c|}{ Vitamin Usage } & \multirow[t]{2}{*}{ Total } \\
\hline & Yes & No & \\
\hline \multicolumn{4}{|l|}{ Treatment } \\
\hline \multicolumn{4}{|l|}{ Chemotherapy } \\
\hline Count & 33 & 129 & 162 \\
\hline$\%$ within treatment & 20.4 & 79.6 & 100.0 \\
\hline$\%$ of total & 16.5 & 64.5 & 81.0 \\
\hline \multicolumn{4}{|l|}{ Chemoradiation } \\
\hline Count & 9 & 29 & 38 \\
\hline$\%$ within treatment & 23.7 & 76.3 & 100.0 \\
\hline$\%$ of total & 4.5 & 14.5 & 19.0 \\
\hline \multicolumn{4}{|l|}{ Total } \\
\hline Count & 42 & 158 & 200 \\
\hline$\%$ within treatment & 21.0 & 79.0 & 100.0 \\
\hline$\%$ of total & 21.0 & 79.0 & 100.0 \\
\hline
\end{tabular}

ation (especially weakness) by using vitamins and supplements, and 16 of them (39\%) reported symptom improvements.

\section{Discussion}

There are a few indications for prescribing vitamins and supplements during the course of cancer treatment. For instance, patients who have undergone gastrectomy require vitamin $B 12$ and iron, or patients who receive pemetrexed need B12 and folic acid supplements. Apart from these exceptions, there is no reliable evidence that any dietary supplement and vitamin can help prevent or treat any type of cancer or its complications. Nonetheless, it seems that many cancer patients tend to use these supplements and little data are available on prevalence of their consumption. Besides, the impacts of nutritional supplements on therapeutic effects of chemotherapy and radiotherapy and their related toxicities are not clear. Our study evaluated the prevalence of taking different types of vitamins and supplements in patients who were in their first three months of treatment.

A cross-sectional survey of 603 cases with common cancers (breast, colorectal, lung and prostate) was conducted for 2 years by researchers at the University of North Carolina Comprehensive Cancer Center. Their results showed that $52 \%$ of the patients during chemotherapy and $51 \%$ of the cases during radiotherapy took vitamins and similar supplements. 


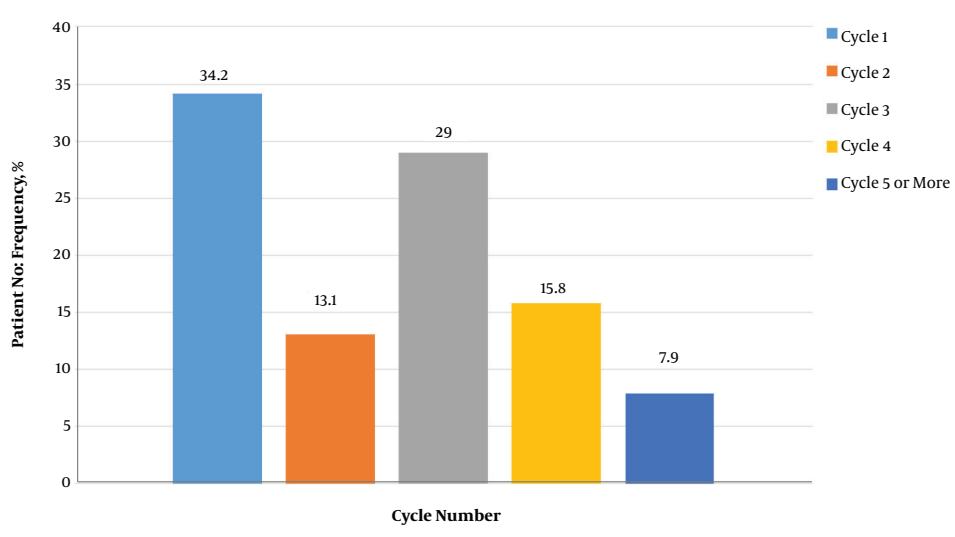

Figure 1. Number of chemotherapy cycle in which patients started vitamin use.

About 75\% of the cases reported professional sources for prescribing supplements; and female patients and those with higher education levels tended to use vitamins more readily (4). Similar to our results, they showed that educated patients were more willing to take vitamins and supplements.

In a systematic review of 32 published studies addressing vitamin and mineral supplement use among US adult cancer patients and survivors in $2008,14 \%$ to $32 \%$ of survivors mentioned starting supplement use after cancer diagnosis.

Breast cancer patients reported the highest use; whereas the least use belonged to patients with prostate cancer. Vitamin and supplement use was more common among female patients and individuals with higher level of education. The interesting point was the fact that about $68 \%$ of physicians were unaware of vitamin use among their cancer patients (2).

While it is known that cancer and its treatments can have adverse effects such as anemia, it was interesting that only 7 out of 29 anemic patients were receiving treatment for this reason, and two others used iron supplements without prescription.

Anemia can affect treatment results, especially in certain malignancies such as cervical cancer. However, only two out of six patients with this disease were receiving appropriate treatment. This shows that many physicians probably pay little attention to patients' comorbidities and treatment adverse effects (5).

Production of reactive oxygen species (as happens in radiotherapy) and generation of free radicals by chemotherapy drugs (by alkylating agents, platinum and antitumor antibiotics) are among main mechanisms by which cancer treatment modalities exert their cytotoxic effect. There is some evidence that while antioxidants may protect normal cells from the oxidative damage caused by chemotherapy or radiotherapy (and hence reduce the adverse effects of treatment), non-enzymatic exogenous antioxidants such as vitamins, minerals, and polyphenols may protect tumoral cells from the cytotoxic effects of cancer treatment. Although the risks and benefits of antioxidant supplementation are still controversial, a recent meta-analysis concluded that the harm caused by antioxidant supplementation remains unclear for patients during cancer therapy (6).

There are some controversies about the efficacy of supplements on chemotherapy or its adverse effects. Branda et al. in 2004 evaluated the effects of vitamin B12, folate, and dietary supplements on chemotherapy-induced mucositis and neutropenia in 49 patients with breast cancer. They concluded that chemotherapy-induced neutropenia might be ameliorated by dietary supplementation with a multivitamin or vitamin E, while high serum folate levels can exacerbate neutropenia. No significant association between oral mucositis and nutritional supplements was found (7).

A cross-sectional, population-based survey compared supplement use among patients with cancer and those with other chronic conditions. Participants reported use of multivitamin, minerals, herbs, and other natural products during the preceding 12 months. The results showed that patients with cancer or other chronic conditions tend to use vitamins and other supplement more frequently than healthy individuals do. Supplement use was similar between cancer survivors and cancer-free individuals correlated by gender (more in women), age (more in advanced age), greater physical activity, and fruit and vegetable intake (8). It seems that female gender is an independent fac- 
tor for taking supplements, regardless of the health condition.

A Turkish study evaluated the prevalence of complementary and alternative medicine (CAM) use in patients who visited the outpatient clinic in the medical oncology department. CAM use included pharmacologic agents like vitamins, dietary supplements, and herbal products or non-pharmacologic methods such as prayer, meditation, hypnosis, massage, or acupuncture. Of 1499 cases who filled the survey questionnaire, 1433 (96\%) reported using of non-pharmacologic CAM. The most commonly used methods were prayer $(n=1,433)$ and taking herbal products $(n=42)$. There were only 60 cases $(4 \%)$ who used pharmacologic CAM and it was not associated with age, gender, or income level. However, these were significantly related to level of education and employment status (9). In this study, age or gender did not influence the use of supplements, which was in contrast to the results of our study.

There is a meta-analysis from the U.S. Preventive Services Task Force which included 19 randomized clinical trials (RCTs) (3 for cancer and 16 for fracture outcomes) and 28 observational studies (for cancer outcomes). Although the data from RCTs showed that vitamin D supplementation may reduce cancer risk, the data from observational studies showed the opposite. The authors concluded that vitamin D and calcium supplements may reduce fracture risk especially in cases who are institutionalized, but the effects of vitamin D in cancer remained elusive (10).

In a study on 241 patients, it was shown that chemotherapy-induced toxicity did not depend on whether the patients were vitamin D depleted or had sufficient levels (11). This study showed that there was some evidence that vitamin D supplements might not be effective in cancer patients.

Another study reported that $20 \%-80 \%$ of individuals used dietary supplements after a cancer diagnosis, and patients with certain types of cancer (breast, prostate, colorectal, and lung) were more willing to take these supplements. The reasons for using supplements were reported as improving quality of life, reducing symptoms related to treatment, prescription by medical practitioners, and suggestions by family and friends (12). These findings are similar to the results of our study.

Song et al. calculated the amount of nutrients consumed from foods and supplements, the percent contributions of supplement nutrients to total nutrient intakes and cancer survivors' nutrient intakes relative to the estimated average requirements (EARs) and the tolerable upper intake levels (ULs) among 400 cancer survivors and 10387 cancer-free individuals, aged $\geq 19$ years in Korea. It was reported that $33.3 \%$ of cancer survivors and $22.1 \%$ of cancer-free individuals used dietary supplements. It was revealed that cancer survivors used more riboflavin, folate, and iron from foods $(\mathrm{P}<0.05$ for each $)$, and also had higher intakes of calcium $(\mathrm{P}=0.05)$ and vitamin $\mathrm{C}(\mathrm{P}=0.01)$ from foods and supplements than cancer-free individuals. In female cancer survivors, those with higher education level, moderate physical activity, low vegetable intake, and high circulating vitamin D levels had higher supplement intake (13). This study shows that there is a trend to higher level of supplements intake in cancer cases. As we do not know the exact effects of various vitamins on oncologic treatment, it is important to ask the patients about supplement use in taking medical history.

\subsection{Conclusions}

In our study, more than $20 \%$ of the patients took vitamins regularly. In more than $40 \%$ of these cases, vitamin supplements had been prescribed by the treating physician. Because of the potential negative effects of vitamins on treatment efficacy, it would be prudent to avoid prescribing them for patients under active cancer treatment. Besides, the treating physician should make the patients aware of these potential hazards.

\section{Footnotes}

Conflict of Interests: The authors declare that there is no conflict of interest regarding the publication of this article.

Ethical Considerations: There were 2 informed consent forms for each case.

Funding/Support: There is no financial support for this study.

\section{References}

1. Miller ER3rd, Pastor-Barriuso R, Dalal D, Riemersma RA, Appel LJ, Guallar E. Meta-analysis: High-dosage vitamin E supplementation may increase all-cause mortality. Ann Intern Med. 2005;142(1):37-46. doi: 10.7326/0003-4819-142-1-200501040-00110. [PubMed: 15537682].

2. Velicer CM, Ulrich CM. Vitamin and mineral supplement use among US adults after cancer diagnosis: A systematic review. J Clin Oncol. 2008;26(4):665-73. doi:10.1200/JCO.2007.13.5905. [PubMed:18235127].

3. Fritz H, Flower G, Weeks L, Cooley K, Callachan M, McGowan J, et al. Intravenous vitamin $\mathrm{C}$ and cancer: A systematic review. Integr Cancer Ther. 2014;13(4):280-300. doi: 10.1177/1534735414534463. [PubMed: 24867961].

4. Luo Q, Asher GN. Use of dietary supplements at a comprehensive cancer center. J Altern Complement Med. 2018;24(9-10):981-7. doi: 10.1089/acm.2018.0183. [PubMed: 30247972]. 
5. Candelaria M, Cetina L, Duenas-Gonzalez A. Anemia in cervical cancer patients: Implications for iron supplementation therapy. Med Oncol. 2005;22(2):161-8. doi: 10.1385/MO:22:2:161. [PubMed: 15965279].

6. Yasueda A, Urushima H, Ito T. Efficacy and interaction of antioxidant supplements as adjuvant therapy in cancer treatment: A systematic review. Integr Cancer Ther. 2016;15(1):17-39. doi: 10.1177/1534735415610427. [PubMed: 26503419]. [PubMed Central: PMC5736082].

7. Branda RF, Naud SJ, Brooks EM, Chen Z, Muss H. Effect of vitamin B12, folate, and dietary supplements on breast carcinoma chemotherapyinduced mucositis and neutropenia. Cancer. 2004;101(5):1058-64. doi: 10.1002/cncr.20484. [PubMed:15329916].

8. Miller MF, Bellizzi KM, Sufian M, Ambs AH, Goldstein MS, BallardBarbash R. Dietary supplement use in individuals living with cancer and other chronic conditions: A population-based study. J Am Diet Assoc. 2008;108(3):483-94. doi: 10.1016/j.jada.2007.12.005. [PubMed: 18313431].

9. Yalcin S, Hurmuz P, McQuinn L, Naing A. Prevalence of complementary medicine use in patients with cancer: A Turkish comprehensive cancer center experience. J Glob Oncol. 2018;(4):1-6. doi: 10.1200/JGO.2016.008896. [PubMed: 30241173]. [PubMed Central: PMC6180833].

10. Wang L, Sesso HD, Glynn RJ, Christen WG, Bubes V, Manson JE, et al. Vitamin E and C supplementation and risk of cancer in men: Posttrial follow-up in the Physicians' Health Study II randomized trial. Am J Clin Nutr. 2014;100(3):915-23. doi: 10.3945/ajcn.114.085480. [PubMed: 25008853]. [PubMed Central: PMC4135500].

11. Kitchen D, Hughes B, Gill I, O'Brien M, Rumbles S, Ellis P, et al. The relationship between vitamin $\mathrm{D}$ and chemotherapy-induced toxicity a pilot study. Br J Cancer. 2012;107(1):158-60. doi: 10.1038/bjc.2012.194. [PubMed: 22588559]. [PubMed Central: PMC3389405].

12. Marian MJ. Dietary supplements commonly used by cancer survivors: Are there any benefits? Nutr Clin Pract. 2017;32(5):607-27. doi: 10.1177/0884533617721687. [PubMed: 28813230].

13. Song S, Youn J, Lee YJ, Kang M, Hyun T, Song Y, et al. Dietary supplement use among cancer survivors and the general population: A nation-wide cross-sectional study. BMC Cancer. 2017;17(1):891. doi: 10.1186/s12885-017-3885-1. [PubMed: 29282002]. [PubMed Central: PMC5745960]. 